\title{
Perspectiva de género en los fenómenos migratorios: estudio desde Europa y América Latina*
}

\author{
Gender perspective in migratory phenomena: a study from Europe and Latin America
}

Yennesit Palacios Valencia**

${ }^{\star *}$ Abogada, PhD en Derechos Humanos y Desarrollo de la Universidad Pablo de Olavide. Actualmente docente-investigadora de la Maestría en Educación y Derechos Humanos, en la Escuela de Posgrados de la Universidad Autónoma Latinoamericana de Medellín Correo electrónico: yennesit.palacios@gmail.com

\section{Resumen}

Este escrito reflexiona sobre la forma cómo la migración impacta en las mujeres, detallando algunos datos que permitan visibilizar a las mujeres como sujetos activos de la migración, pero sin desconocer, al mismo tiempo, el grado de vulnerabilidad en el que están expuestas. Para dicho estudio se contextualizará el escenario europeo, específicamente España, puntualizando su estrecha relación con el escenario latinoamericano, ya que son dos contextos ligados entre sí por razones históricas que convocan a reflexionar sobre el papel de las mujeres, sin desmeritar el papel de los hombres, en estos fenómenos migratorios.

Palabras clave: Derechos humanos, Europa, Género, Inmigrantes, Latinoamérica.
Recepción

15 de septiembre de 2016

Revisión:

10 de octubre de 2016

Aprobación:

18 de noviembre de 2016

\begin{abstract}
This paper reflects on how migration impacts on women, detailing some data that make women visible as active subjects of migration, but without ignoring, at the same time, the degree of vulnerability in which they are exposed. This study will contextualize the European scenario, specifically Spain, highlighting its close relationship with the Latin American context, since they are two contexts linked together for historical reasons that call for reflection on the role of women without demeaning the role of men, in these migratory phenomena.
\end{abstract}

Keywords: Human rights, Europe, Gender, immigrants, Latin America.

\section{Introducción}

Sirva como primer punto de partida el precisar que es un hecho indiscutible que los fenómenos migratorios afectan de manera distinta a hombres y mujeres; y aunque hasta la década de los 80 la migración no daba relevancia a la participación de las mujeres, hoy en día, en la escala mundial, "las mujeres han tenido una gravitación importante en la migración internacional que registra un aumento relativo desde 1960, a pesar de que todavía no son mayoría" (Martínez, 2003, p. 19). Hecho que visibiliza, sin lugar a dudas, lo que los teóricos han llamado la feminización de la migración, no obstante, todavía el debate gira en torno a los hombres como la "norma", ignorando las particularidades de las mujeres en dicho contexto.

Lo esencial para analizar teniendo en cuenta la premisa anterior, es que en el caso de las migraciones y partiendo del género como categoría analítica, las mujeres migrantes pueden estar expuestas a una doble

El escrito se presenta como un artículo de revisión, fundamentado como parte de la Línea de Investigación en Género, Minorías Étnicas y Grupos Vulnerables de la Escuela de Posgrados de la Universidad Autónoma Latinoamericana de Medellín (UNAULA). 
vulnerabilidad, la derivada en consideración al género, otras por factores de edad, sobre todo en el caso de niñas y adolescentes (Torrado, 2012, p.68), y ésta a su vez puede verse deteriorada por factores de discriminación, si aquellas son pertenecientes a minorías étnicas como ocurre con las procedentes de África a Europa, e incluso desde América Latina.

Sustancialmente, dado el consenso acerca de la indiscutible presencia de las mujeres como plantea Sassen (2003, 2003b, 2007), en las migraciones, y por las particularidades derivadas de dicho fenómeno, se ha motivado la búsqueda de cuerpos teóricos que expliquen el impacto de la migración femenina en las sociedades de origen y de destino. En consecuencia, a partir de esta preocupación se han hallado nuevas miradas sobre la movilidad humana femenina (Morcillo, 2012), guiando el análisis sobre las necesidades y carencias existentes, como medidas ineludibles a tener en cuenta en el diseño de políticas públicas en materia migratoria.

Si bien, los flujos migratorios no son nada novedoso, puesto que son tan antiguos como la propia historia humana, es verdad constatada que los flujos y movimientos de personas se apoyan y mutan por el acelerado proceso de integración global, dando lugar a una época que se ha denominado, la era de la migración (Castles y Miller, 2004). Al respecto, se debe entender la cuestión de la migración, siguiendo a Sassen (2007, p. 39), como representación también de lo mundial o lo transnacional; siendo también un elemento esencial como plantea Puyol (2002, p. 56), del funcionamiento de la economía.

En relación a lo anterior, este escrito reflexionará sobre tres puntos específicos y se dividirá de la siguiente manera: la primera, se centra en revisar a partir de estudios realizados sobre la caracterización que surge en relación a las necesidad de migrar, los riesgos y miedos derivados; en cuanto a la segunda, puntualiza en lo que se ha denominado la feminización de la migración, y se concentra en la vulnerabilidad de las mujeres en los fenómenos migratorios; a posteriori, se hace un estudio del caso de Europa y América Latina en relación al impacto de los fenómenos migratorios, pero teniendo en cuenta el contexto colombiano y español.

Finalmente, este escrito permitirá concluir que las políticas migratorias que los distintos gobiernos desarrollan y ejecutan deben tener en cuenta, por antonomasia, la defensa de los derechos humanos, precisamente para coadyuvar en la construcción de pautas que permitan cohesión social lejos escenarios desiguales y excluyentes, para despensar y repensar el imaginario social existente, en el intento de impulsar un cambio de paradigma que implique la transformación del cliché implícito en el discurso "normalizado" que, visibiliza a la comunidad de inmigrantes como un problema, situación que conlleva con frecuencia, contextos de exclusión social. Imaginario propiciado también por el papel que desempeñan los medios de comunicación en general y las noticias en particular, en la reproducción de la desigualdad «racial» y étnica (Dijk, 2005, p. 33), en las sociedades receptoras de inmigrantes.

\section{La necesidad de migrar, los riesgos y miedos derivados}

La inmigración en sus diferentes manifestaciones se proyecta como un fenómeno mundial y complejo, constituye un fenómeno social de primera magnitud en el mundo contemporáneo (Blanco, 2006, p. 7), con diversas causas y variaciones. Dichas divergencias dependerán en buena medida si el análisis se hace vinculando a las personas migrantes en el marco de la legalidad o "ilegalidad". Situación que en cualquiera de los dos casos, pero principalmente en el último, vincula a las personas que emigran a terceros países con el enemigo que genera malestar. Leitmotiv que está estigmatizando a los "Otros", los extraños a la comunidad.

Todo ello está también ligado a la proliferación de "organizaciones criminales dedicadas al tráfico de inmigrantes "ilegales" que cooperan a reforzar la emigración de las personas provenientes de los países económicamente 
menos favorecidos" (Serrano, 2002, p.314). Elemento que complejiza el hecho mismo de emigrar, siendo una coyuntura que sitúa a la población inmigrante con alto grado de vulnerabilidad.

Lo anterior tiene serias implicaciones, en tanto ese "Otro" termina siendo excluido y se convierte no sólo en el mal que hay que combatir, sino en una carga para el sistema, hecho que motiva el diseño de duras políticas de cierres fronterizos. Sin embargo, los fenómenos migratorios se extienden al tiempo que aumentan las políticas de cierres fronterizos, contrasentido que desvirtúa la naturaleza y el sentido social de migrar. Por algo se dice que "los humanos no tenemos raíces sino pies: no estamos sujetos a la tierra como los árboles, sino que podemos viajar de un lado para otro y asentarnos en el lugar que nos parezca más habitable". "Nuestra propia constitución anatómica pone de manifiesto que estamos hechos para andar y correr erguidos a través de largas distancias: el homo sapiens es un homo viator" (Campillo, 2005, p. 107).

En general, si se observa el contexto europeo (Kymlicka, 2001, p. 18-19), parece manejar una política de antiinmigración, la prioridad parece ser que los no comunitarios o ciudadanos de segunda, no lleguen, o, en caso tal, lleguen a territorio europeo pero no para quedarse. Idea que es reproducida, popularizada y legitimada por las diferentes esferas institucionales, las cuales se ven expresadas en las normativas que evolucionan para reaccionar ante las personas no gratas a la comunidad. En efecto, la inmigración es particularmente interesante para ilustrar las políticas gubernamentales de "antidelincuencia", pues la xenofobia permite descargar la ansiedad social en un objetivo fácil y de bajo costo:

Ya lo han experimentado intensamente desde hace tres décadas los países de Europa, con los planteamientos ultranacionalistas del Frente Nacional de Jean-Marie Le Pen, en Francia, o de dirigentes de ultraderecha como el asesinado Pim Fortuyn, en los Países
Bajos, Jörg Haider, en Austria, Pia Kjaersgaard, en Dinamarca, Umberto Bossi, en Italia o muy recientemente Nikolaos Maichaloliakos, en Grecia, quienes, entre otros, han desempeñado exitosas campañas de ley y orden vinculando el fenómeno de la delincuencia con el de la inmigración (Horvitz, 2012, p.103).

No es un hecho desconocido que en los países desarrollados la inmigración es un tema repudiado y de preocupación social, y cuando se habla de inmigración parece como si sólo fuesen inmigrantes los procedentes del continente africano y latinoamericano, análisis que vincula la pobreza con la migración. Es más, ser inmigrante en el contexto europeo se reduce normalmente, a ser "moro» o "negro» (Vargas, 1998, p. 61). No obstante, son estereotipos marcados que desconocen que incluso las sociedades que "mejor resistieron el auge de la marginalidad avanzada, como los países escandinavos, se ven tocadas por este fenómeno de estigmatización territorial ligado a la aparición de zonas reservadas a las parias urbanas" (Wacquant, 2007, p. 194).

Estos elementos descritos conllevan diversas implicaciones para la inmigración en la era de la globalización. Porlotanto, expertosenla materia, desde economistas, juristas, antropólogos, sociólogos y demógrafos, entre otros, se han dedicado a estudiar con arduo detenimiento, cómo es el proceso de incorporación de las personas migrantes no sólo en el marcado laboral, sino su incorporación misma en las sociedades de acogida. Preguntas como quién migra, porqué se migra, son interrogantes que siguen siendo estudiados, integrando también la pregunta sobre cuál es el papel, en particular, de las mujeres, problematizando en las cuestiones económicas, políticas, sociales y culturales que crean este fenómeno (Castillo, 2013, p. 66).

Es de resaltar que la complejidad de la migración internacional que aumentó desde los años noventa, "mantiene una visión conflictiva de la inmigración y se realza la oportunidad que 
ofrece la emigración para las sociedades de origen y las personas migrantes, no sin riesgos que amenazan sus derechos humanos y la erosión de masas críticas de capital humano" (Martínez, 2003, p.11), dado que se subvaloran las aportaciones derivadas de los colectivos que migran a terceros países. Sin exclusión, en algunos escenarios, que la población de inmigrantes sea apetecida, pero en función de las necesidades de los países de acogida; bien por políticas que necesitan luchar contra el envejecimiento que amenaza la existencia humana a largo plazo, o bien, por la necesidad de mano de obra barata que haga las labores que otros no desean.

\section{Miedos y riesgos: a propósito de los este- reotipos contra la población de inmigrantes}

Los estudios demuestran que las migraciones también se ven inundadas, por niños y niñas solos/as, y sin referente familiar adulto que les acompañe, en cuyo caso no están asociados única y exclusivamente a los flujos de explotación laboral, sino sexual (Torrado, 2012 , p. 69). Todo estos factores se agravan por el discurso altamente estereotipado, donde los y las inmigrantes son vistos como los extraños con derechos fuertemente limitados, al convertirse en una categoría de sujetos regidos bajo un régimen de excepcionalidad sin garantías (CEAR, 2009, p. 26), y en el caso particular del inmigrante "ilegal" (Valero, 2005 , p. 116), sin papales (Vite, 2006, p. 18), automáticamente pierden sus derechos. Base soportada en las mismas políticas migratorias, ya que están exactamente diseñadas para que aquellos no gocen de ciertas garantías, pues se infunde terror colectivo bajo la excusa de la "ilegalidad" y así, sus derechos no son reconocidos. Circunstancia que termina por empeorar, a gran escala, los efectos del hecho mismo de emigrar, por la carencia de unas condiciones idóneas que permitan satisfacer unos estándares mínimos de bienestar.

Fundamentalmente los/as inmigrantes son en su mayoría personas de pocos ingresos, procedentes de los países menos desarrollados, con niveles de educación bajos o medios, y con la disposición a aceptar trabajos que nadie desea (Sassen, 2006, p. 19). En este contexto aparecen los/a inmigrantes como los condenados de la tierra, -a propósito de la tragedia Lampedusa en Italia- que como expresa Naïr, "huyen del hambre y de las guerras civiles, y que estafados por los contrabandistas clandestinos y de las magias del éxodo, no encuentran al término de su recorrido, [cuando no la muerte], y más que la cárcel, los centros de internamiento, las expulsiones brutales, y cuando logran pasar entre las estrechas mallas de las redes de acero construidas por los países de acogida, desembocan en la miseria de la clandestinidad y de la vida sin derechos" (4 de octubre de 2013). El drama es siempre el mismo, mujeres algunas veces embarazadas, niños, niñas y jóvenes de todas las edades, pensando que emigrar de su país de origen será mejor que resignarse a morir en él.

Lo paradójico en este contexto es que los países de acogidas no son necesariamente la respuesta anhelada, pues dada la precariedad en la que están insertos por estar en la clandestinidad -sobre todo si se vive en condición irregular- pueden llegar a padecer en carne propia también el drama de la discriminación, viéndose obligados a soportar la tacha de la élite social que los estigmatiza como los "Otros". Es de destacar en estos casos, que el papel de las mujeres se ve aún más afectado, dado los escenarios de discriminación producto de un sistema de subordinación y dominación entre hombres y mujeres. En suma, el trabajo informal, el trabajo ilegal y la emigración se presentan como formas cada vez más importantes para garantizar la subsistencia (Sassen, 2003b, p. 17), pero bajo fórmulas que no son necesariamente las más garantistas.

Es inevitable hablar de los fenómenos migratorios sin ligarlo a la búsqueda de salida a la pobreza -el Informe sobre Desarrollo Humano 2013, constata que alrededor de 1,57 mil millones de personas, o más del $30 \%$ de la población de los 104 países analizados, viven en condiciones de pobreza multidimensional- 
esto es una de las causas de la emigración, pues ante el desespero de salir de la miseria, migrar a todo costa termina siendo la primera opción.

Con estos presupuestos la configuración de los movimientos migratorios internacionales se puede explicar acudiendo a factores predisponentes y precipitantes, o en términos de atracción y de expulsión en los términos de Torrado (2012, p.68). En el último evento, destaca como un elemento fundamental el hecho de vivir en la pobreza y en desempleo (Sassen, 2007a, p. 167); y entre los elementos de atracción opera la posibilidad de obtener un empleo y una mejor remuneración. Aun así, muchas científicas sociales creen que para analizar las migraciones desde una perspectiva de género, es necesario tener en cuenta diversos aspectos que van desde el ámbito familiar y los contextos sociales, hasta la estructura familiar -número de hijos/as, tamaño de la familia, roles- (Torrado, 2012, p. 69).

No obstante, lejos de llegar a dignificar la existencia con el hecho mismo de emigrar, independientemente de que se trate de hombres o mujeres, las ofertas encontradas como medios de subsistencia suelen estar asociadas en gran proporción con puestos de trabajo tipo manual y poco remunerado, muchos de ellos ocupados por mujeres e inmigrantes (Sassen, 2007b: 38). En este contexto como explica Javier de Lucas $(2006$, p. 3), el desafío de la inmigración es mucho más importante que la dificultad de acomodar estadísticas y recursos en relación con las necesidades del mercado laboral, o las medidas a adoptar para mantener el standard de orden público. Al respecto dice el autor:

Hay que mirar más allá y entender que los flujos migratorios son el catalizador que nos sitúa ante la necesidad de volver a pensar a radice nuestras respuestas a la construcción democrática del vínculo social y político, porque estas comportan hoy un déficit de inclusión y pluralidad en la medida en que institucionalizan la exclusión de los inmigrantes como sujetos del espacio público, y su imposibilidad conceptual de llegar a ser ciudadanos (2006, p. 3).

A este panorama pueden agregarse diversos riesgos procedentes de las sociedades receptoras de inmigrantes, especialmente en las sociedades más ricas suelen registrarse, con frecuencia, reacciones de resistencia por parte de sectores que, manejando no siempre datos contrastados, sienten amenazado su propio bienestar material (Velasco, 2010, p.585-586). Así, se ha popularizado la visión de la inmigración como fenómeno masivo, que atenta contra la seguridad ciudadana y contra la identidad nacional; vinculan con su llegada todos los males del país: "pérdida del bienestar social, inseguridad, delincuencia, desempleo; plantean la dicotomía entre un pueblo que no los quiere y unos políticos, o unos capitalistas, según los casos, que los utilizan en su beneficio" (Valero, 2005, p. 116).

De hecho, la violencia y la amenaza están entre las principales propiedades del significado del discurso periodístico sobre inmigrantes. Sin embargo, la violencia y la amenaza no radican meramente en el hecho de que algunos individuos lleguen al país, sino que se sugiere entonces, que llegan masivamente y de manera organizada como si se tratara de un ejército (Dijk, 2005 , p. 46). Además, ante el boom publicitario de la seguridad ciudadana, que relacionan al inmigrante con la delincuencia, estampa en aquellos no sólo la etiqueta de la marginalidad, sino la cruel consecuencia, como expresa Zaffaroni (1994, p.87), de ser el "diferente", recién llegado, y por ende, más desconocido. Por ello, se ha advertido la dualidad entre el extranjero que aparece como cicatriz entre los seres humanos y los ciudadanos, en razón a que la figura de los y las inmigrantes:

no es ya la de lo exótico que nos fascina, ni la del buen salvaje originario y naturalmente puro, ni la del alter ego del filósofo que posibilita la apertura a otras culturas y la mirada autocrítica, ni la del romántico "otro" misterioso y seductor, ni la de los pioneros que se 
arriesgan en la conquista de un territorio y hacen pueblo, ni siquiera la de los Gastarbeiter (trabajador inmigrante), llamados para colaborar en el desarrollo, sino la del intruso que irrumpe en nuestra casa común y perturba el buen orden de nuestra sociedad acomodada. Su aparición es intempestiva, su presencia con frecuencia ilegal y su inscripción problemática (Fernández, 1998, p. 100).

En definitiva, aparece un nuevo racismo, que en nada tiene que ver con los elementos clásicos. En el nuevo racismo, las minorías no son biológicamente inferiores, sino diferentes; a veces observados con sospecha de «patologías» que, por supuesto, deben ser corregidas (Dijk, 2005, p. 34). Dicho de otra forma, quien ostenta el calificativo de inmigrante, y peor aún, si está indocumentado, no saldrá para nada bien librado.

Por otra parte, un análisis con perspectiva de género, como se observará más adelante, vislumbra que con frecuencia se cometen graves violaciones a los derechos humanos de las mujeres, lo cual no significa que los hombres también sean afectados, en el entendido que: "la clandestinidad llega a ser uno de los factores determinantes de la explotación sexual de inmigrantes obligadas a prostituirse. Cuya absoluta indefensión les impide una resistencia eficaz frente a las redes de tratas de personas. Recordando además, que según fuentes concordantes, el porcentaje de mujeres inmigrantes que ejercen la prostitución supone más del $90 \%$ del total" (Terradillos, 2006, p. 53). Lo preocupante es que la persecución y afectación en los derechos será una presencia hereditaria, pues además de catalogar a personas como inmigrantes -término que arrastra un lastre peyorativo- (García, 2003, p. 29), sus descendientes a su vez, heredarán la etiqueta.

Todos estos aspectos terminan por desconocer que "la inmigración y la incorporación de minorías étnicas y raciales son las dos fuentes más comunes de diversidad cultural en los Estados modernos. Lo cual hace necesario diseñar y formular nuevas políticas multiculturales que propicien una mejor integración sociocultural y socioeconómica" (Aguilera, 2012, p. 924), para afrontar las dinámicas que envuelven el desarrollo de una manera deshumanizante, y hacer exigencias como críticos de un enfoque de derechos desde abajo, visibilizando las necesidades existente en estos escenarios de exclusión y pérdidas de derechos.

\section{Feminización de la migración}

La situación de inequidad, desigualdad social, económica y política, que viven las mujeres es un hecho evidente en la historia de la humanidad. Aunque afirmar tal cosa no deja de ser pura obviedad, siendo una cuestión rigurosamente investigada, analizada y reconocida a nivel internacional, regional, nacional y local, que se expresa permanentemente en la constante vulneración de sus derechos humanos y en la aceptación sociocultural de la violencia contra las mujeres, además de las desventajas en el disfrute de los beneficios del desarrollo, por mencionar algunos aspectos, es una situación que perdura en la época actual.

No obstante, aún la historia marcada por la desigualdad entre hombres y mujeres en los distintos ámbitos, desde lo familiar hasta lo laboral, las transformaciones derivadas del paso a una "aldea global" siguen marcando la pauta en relación a las dinámicas históricas de género y raza en el desarrollo capitalista, apareciendo diversos fenómenos, entre los que se destacan, la feminización de la pobreza y la feminización de la fuerza de trabajo (Sassen, 2003b, p.17), que con distintos rasgos se han incrementado a lo largo de las últimas tres décadas en los países hegemónicos y periféricos.

Esto ha dado lugar, según explican los demógrafos, a estudiar también la feminización de las migraciones como parte del fenómeno más amplio de la 'globalización de las migraciones' (Pérez y Paiewonsky, 2008, p. 34), y aunque no es el único factor derivado de dicha relación, existe un nexo necesario entre migración y desarrollo; apareciendo como 
un aspecto de suma importancia el concepto de "remesas para el desarrollo" (Petrozziello, 2003, p. 19).

Las remesas, entendidas como los recursos que los emigrantes envían a sus países, normalmente a sus familiares, así como las inversiones personales y las donaciones privadas, se encuentran entre "las principales actividades económicas transnacionales a las que se dedican los/a migrantes y sus familias. Las remesas, de hecho, equivalen a casi el 15 por ciento del PIB de muchos países" (Orozco, 2014 , p. 5). Lo clave a destacar en este contexto es, que en la última década, se ha propagado una presencia significativa de las mujeres (Cfr. Sassen, 2003b, p. 41), en una gran variedad de circuitos transfronterizos.

El género, introducido en los modelos empíricos de investigación, se convierte de este modo en una variable relevante a la hora de explicar las formas de la migración y sus resultados (Molpeceres, 2012, p. 93). Al respecto, en los últimos años el término "feminización de las migraciones" se ha vuelto un tema de debate, pero el término puede confundir en tanto sugiere un incremento absoluto en la proporción de mujeres migrantes, cuando de hecho, hacia 1960 las mujeres ya representaban el $46.8 \%$ del total de migrantes internacionales, cifra que crecería menos de tres puntos durante las siguientes cuatro décadas, hasta alcanzar el $49.6 \%$ en el 2005 (Pérez y Paiewonsky, 2008, p. 34).

Si bien, en este panorama el tema de las remesas adquiere un peso representativo, dada la creciente teorización sobre sus beneficios y virtudes, en la evaluación macroeconómica y microsocial de sus impactos, también es cierto como expresa Martínez Pizarro (2003, p. 18) que, nadie que esté familiarizado con el campo de las remesas podrá desconocer que existen diversas preguntas, con respuestas pendientes, que apuntan entre otras, a las formas de "reducción del costo de transferencias, el monto y modalidades involucradas en los envíos informales, los efectos de dependencia macroeconómica y sobre los hogares receptores, el papel, eficiencia y disciplina de las mujeres vis-à-vis con los hombres, como emisoras y receptoras de remesas, o la sostenibilidad de esta fuente de recursos en ausencia de iniciativas de apoyo a las diásporas" (2003, p. 18).

Pero sobre lo que si hay claridad es, sobre la alta participación de las mujeres en estos procesos migratorios, pues han transformado las tendencias, se han reconfigurado, situándose con un papel activo en el desarrollo global, lo cual permite hacer una diferenciación sustancial en el efecto de las migraciones desde una perspectiva de género.

Así, aunque la distinción entre los impactos económicos en relación al género marca la pauta en los fenómenos migratorios actuales, no se puede desconocer que distintos grupos étnicos, y de diferentes condiciones económicas, sociales y edades, cruzan fronteras en busca de mejores condiciones de vida, nuevos horizontes laborales, ante la urgencia muchas veces, de enviar dinero para el sostén de sus familias. Sin embargo, este análisis llama la atención en la participación activa que vienen tomando las mujeres, pues se calcula que un tres por ciento de la población mundial -cerca de 200 millones de personas- vive fuera de sus países de origen, $50 \%$ de los cuales son mujeres (Pérez y Paiewonsky, 2008, p. 4).

Siendo indiscutible que el enfoque de género recorre estructuralmente las decisiones, trayectorias y consecuencias de la migración (Martínez, 2011, p. 8); como por ejemplo, la decisión de qué miembro de la familia migra, la inserción laboral en el país de acogida, la frecuencia y uso en el envío de remesas (Pérez y Paiewonsky, 2008, p. 4), entre otros. Así entonces, éste hecho es el que empieza a llamar la atención de teóricos y expertos en la materia, dada la necesidad de hacer un análisis obligado que distinga la capacidad de salida del lugar de origen, el efecto de la llegada, motivaciones y de más, que impactan de modo diferente, haciendo que las mujeres influyan, esencialmente, en los nuevos modelos que están marcando la relación costo-beneficio de los fenómenos migratorios, en una época 
donde el capitalismo se funda como patrón en los fenómenos ligados a la globalización financiera y comercial, y no tanto fronteriza.

Con razón se dice que "la mundialización rompe todas las fronteras, somete a todas las sociedades, transforma a los Estados en guardaespaldas del capitalismo financiero, abre de par en par las arterías de circulación a las mercancías, a los capitales, a la comunicación, y a las personas que tuvieron la suerte de nacer en el lugar adecuado..." (Naïr, 2010, p. 430). Pues no hay duda que, aún la llamada época de la globalización, se afronta un período de endurecimiento de leyes migratorios y de cierre fronterizo, situación que está influenciada por el discurso estereotipado que excluye a las poblaciones de inmigrantes.

De todas formas, es necesario precisar que la situación de las mujeres, en particular, se ve afectada de manera específica y distinta, es más, dentro del grupo de mujeres que emigra, según el punto de partida, existirán al mismo tiempo diferentes grupos de mujeres, con características culturales y necesidades distintas, justamente producto de las diferencias culturales. Con lo cual, éste es otro punto que marca un aspecto crucial en el debate, pues el hecho de ser inmigrante, mujer, y con connotaciones culturales disímiles, conlleva una serie de impactos que afectan a las mujeres según el lugar de procedencia y de destino.

Por otra parte, los factores de empuje a sociedades desconocidas, pero que llaman la atención al ofrecer, en apariencia, mejores oportunidades de vivir dignamente, también se ve afectado por el rol patriarcal marcado a lo largo de la historia, situación que también pone en desventaja y en detrimento las oportunidades existentes en el mercado laboral. Por ende, no puede desconocerse el grado de vulnerabilidad en el que se ven inmersas las mujeres desde el momento mismo que abandonan el seno familiar: la ruta marcada para salir a otro país, los estereotipos sociales, las redes de tratas de personas, la prostitución, la explotación sexual y laboral, además del abaratamiento de la mano de obra dentro de la posición de inferioridad que ocupan las mujeres en el mercado laboral, son factores que deterioran la vida de cualquier persona que abandone su país de origen en busca de oportunidades y de un mejor vivir con dignidad.

\section{La vulnerabilidad de las mujeres en los fenómenos migratorios}

Como se mencionó, las mujeres migrantes están expuestas a riesgos mayores en cuanto a discriminación, explotación y violencia, ya sea durante sus travesías o en los lugares de destino. En efecto, en lo que respecta al tráfico ilegal de mujeres (Bonelli y Ulloa 2001, p. 61), y de niños y niñas para la industria del sexo (cfr. Defensor del Pueblo, 2012, p. 29), ${ }^{1}$ están creciendo en importancia como formas de obtención de ingresos (Sassen, 2003b, p. 41).

Ser inmigrante y mujer, es una pieza sustancial para analizar el impacto de los fenómenos migratorios, ya que exige reconocer la realidad ambivalente, jurídica y asimétrica de las mujeres en todo un entorno conflictual derivado del ritmo acelerado de cambios que emergen en el eje planetario. Siendo imperioso regularizaciones más garantistas que aboguen por un respeto mínimo de derechos derivados de la propia condición del género humano.

Dicha confluencia está relacionada con algunas de las principales dinámicas de la globalización: "la formación de mercados globales, la intensificación de redes transnacionales y translocales y el desarrollo de tecnologías de la comunicación que eluden fácilmente las prácticas convencionales de control" (Sassen, 2003b, p. 41), y la ficción de la distancia existente entre las fronteras, pues el avance tecnológico y comunicacional hace cada vez más cercana la relación entre los que emigran y sus familiares.

$1 \mathrm{Al}$ ser objeto de denuncias más frecuentes, la explotación sexual ha pasado a ser el tipo de trata más documentado en las estadísticas globales. En cambio, otras formas de explotación o son notificadas suficientemente: el trabajo forzado o en condiciones de servidumbre; la servidumbre doméstica y el matrimonio forzado; la extracción de órganos; y la explotación de los niños[a] en la mendicidad, la industria del sexo y la guerra (2012: p. 29). 
Aunque lo anterior es una importante ventaja para la entrada y salida, desde y hacia otros países, es una gran desventaja por la instrumentalización del ser humano producto de la dinámica entre oferta y demanda, en relación a la confluencia del género, etnia, nacionalidad e indocumentación, que pueden conducir a las más extremas violaciones de derechos humanos, incluyendo abusos sexuales, deterioro de la salud reproductiva y amenaza a la integridad física. En específico, las mujeres integran el grupo más alto de víctimas de engaño por organizaciones dedicadas a la trata de personas con fines de explotación, razón por la cual se encuentran entre las más vulnerables (Martínez, 2003, p. 8).

Desafortunadamente, las mujeres forman parte del grupo de mayor importancia en los sectores de la industria del sexo y se están convirtiendo en un grupo mayoritario en la migración derivada por la búsqueda de empleo. El uso de mujeres extranjeras cubre una amplia gama en crecimiento de sectores económicos, algunos ilegales - verbigratia la prostitución- (Instituto de la Mujer, 2009, 25). Asimismo, aunque es factible que las migrantes a la llegada encuentren algún empleo relacionado con labores domésticas, éste también puede estar relacionado con aspectos tales como: "la agricultura donde sus sueldos, que son relativamente menores, representan una ventaja comparativa. En estos sectores, las condiciones de trabajo son a menudo de explotación y el empleo es informal y carece de seguridad, siendo apetecibles mujeres jóvenes, flexibles y baratas" (Franck y Spehar, 2010, p. 3), en suma, teniendo en cuenta tales circunstancias, las mujeres en este contexto son vistas como sujetas sin derechos.

Sin embargo, lo anterior no quiere significar que las mujeres migrantes no estén cualificadas para ocupar otros cargos y empleos, pero muy posiblemente la vía rápida para adquirir ingresos sea devaluar su propio talento humano para poder subsistir, y en buena medida, enviar parte de su salario a algún destino relacionado con el lugar geográfico donde habitan sus familias, hecho que da relevancia como se mencionó, en acápites anteriores, a las llamadas remesas.

Dentro del marco europeo, por ejemplo en España, "la construcción, la agricultura, la ganadería, la hostelería, y las empleadas del hogar, son cuatro sectores donde la mujer inmigrante tiene mayor presencia, tanto las legales como las ilegales" (Sánchez, 2010: p. 233). Pero si se observan las evaluaciones de riesgos laborales de estos sectores, dan cuenta que existen los llamados riesgos genéricos que afectan a todos los trabajadores sin excepción, como pueden ser: "carga física, condiciones ambientales, riesgo de manejo de maquinarias, riesgos químicos o biológicos, ergonómicos, (...) quemaduras, golpes, cortes, ruidos, sobreesfuerzos, lesiones, etc." (Sánchez 2010 , p. 233). En este caso, como muy bien se explica en el informe La migración laboral de las mujeres en el contexto de la globalización (Franck y Spehar, 2010: 3), en el área de trabajo doméstico las trabajadoras son extremadamente vulnerables a la explotación, pues muchas migrantes trabajan sin papeles o sin un contrato en regla. Existiendo numerosos ejemplos sobre abusos, jornadas de trabajo excesivas, salarios bajos y falta de protección legal.

Dicho de otra forma, un trabajo bien remunerado y las condiciones derivadas de las prestaciones sociales no son las más idóneas si se carece del estatus de inmigrante documentado, o bien, teniendo dicha calidad, el uso abusivo de las empresas en detrimento de quienes desesperados llegan en busca de trabajo, hace que se llegue por tendencia al abaratamiento exagerado de la mano de obra, lo cual da poco, o ningún crédito, a aquellas personas que cuentan perfectamente con cualificación para ejercer incluso cargos de dirección y profesiones importantes, siendo esta desventaja de manera implícita, una consecuencia que impacta, y muy particularmente en las mujeres. 


\section{Vulnerabilidad derivada de los Centros de Internamientos}

Sobre el particular, si el estudio se centra en aquellas mujeres que viven en la clandestinidad, investigaciones sobre el tema han dedicado una central atención a estudiar qué pasa con aquellas mujeres que viven indocumentadas. La respuesta inmediata será que pueden terminar en los Centros de Internamiento (CIEs). Esto analizado en el ámbito europeo -situado específicamente en España- tiene un mayor impacto, toda vez que España ocupa una posición territorial estratégica y es un país privilegiado por latinoamericanos, dada la posibilidad de fácil comunicación por el idioma, y también por originarios del África subsahariana, precisamente por la posición geográfica respecto de España como la puerta de entrada al continente europeo.

En esta coyuntura los y las inmigrante mal llamados "ilegales" se han convertido en el sujeto escogido para el discurso de la emergencia: "los centros de internamiento para extranjeros, verdaderos agujeros negros del Estado de Derecho, los invernaderos de plástico, donde los inmigrantes son degradados a meros cuerpos esclavizados por la agroindustria, los miles de prostíbulos, en los que las inmigrantes son retenidas y explotadas sexualmente... Son lugares todos donde la reducción de seres humanos a mero cuerpo, revela la significación biopolítica de las políticas de inmigración" (Zamora, 2005, p. 152) como constante. Por ende, la "penalización" del cruce fronterizo es la solución al manejo de los "sin papeles" a través de los CIEs.

Aquellos han aparecido en toda Europa para internar a inmigrantes que han recibido una orden de expulsión y están a la espera de su retorno. No son centros penitenciarios, y los que son privados de libertad en ellos no han cometido delito alguno (Zamora, 2005: p. 153); operan básicamente para la detención y custodia de extranjeros sujetos a un expediente de expulsión. Por tanto, la finalidad declarada de los mismos es meramente preventiva y cautelar (Silveira 2002, p. 93).
La realidad demuestra, por tanto, que la expansión normativa apunta al "reforzamiento de los procedimientos de devolución, así como a la regulación de la estancia en los Centros de Internamiento por parte de los extranjeros" (Vicente, 2005: 203). Con todo, la orden de expulsión y la puesta en libertad, terminan siendo en realidad la expulsión del sistema de garantías jurídicas al situarlos en una ambiente de total desamparo legal. Por ello podría decirse que estas instituciones actúan de facto como «creadoras de extranjeros a-legales y marginales» (Zamora, 2005, p. 153).

La secuela inmediata es un escenario de flagrantes violaciones de los derechos humanos, pues la construcción del inmigrante/ enemigo como "infrasujeto no persona, ergo como infraciudadano, un status jurídico que se basa en la negación de los principios jurídicos más elementales" (Lucas, 2005, p. 215), donde para los y las inmigrantes por su construcción como extranjero, no valen las reglas del Estado de Derecho al contrario que para el ciudadano.

La justificación de ese status es la "dominación/ subordinación y desigualdad/discriminación, junto a esa visión instrumental del inmigrantes y desigualdad ante el derecho. Pero la forma más fácil de reorientar el debate, es a través de la no criminalización o descriminalización de los delitos de explotación laboral" (Terradillos, 2006: 43); pues aparece un círculo vicioso que se mantiene, no-son personas, pero ayudan siendo no-personas al mantenimiento del sistema, luego entonces, los CIEs, como explica Comisión Española de Ayuda al Refugiado (CEAR, 2009, p. 26) se mantienen bajo la cara de la legalidad del Estado que protege a sus ciudadanos de los extraños, cueste lo que cueste. Esto puede ser confirmado por el informe presentado en 2009 por la CEAR, lectura que habla por sí sola sobre la situación los CIEs en España.

En este orden de ideas, es un hecho notorio que el fenómeno de la inmigración "exige reconocer los centros de internamientos, las legislaciones y políticas de excepcionalidad, la criminalización de la inmigración sin papeles, etc., como la otra 
cara de la nueva flexibilidad del capitalismo, es decir, como metáforas del control despótico sobre la movilidad de la fuerza de trabajo" (Zamora, 2005, p. 154). Ahora bien, algunos estudios han señalado, en concreto, que en el caso de España la sobrerrepresentación en el CIE de las mujeres relacionadas con el mercado del sexo no es sólo una percepción derivada de estudios en la materia, sino que existen datos oficiales que contrastan dicha información (Martínez, 2013, p. 32).

Los datos también confirman que los países que más están asociados a la trata de seres humanos en España (Defensor del Pueblo, 2012, p. 105), serían: Nigeria, Brasil y Paraguay (Fernández, 2011), junto a Rumania. ${ }^{2}$ No obstante, más allá de los datos oficiales y estadísticos, el riesgo mayor es el que sufren los y las inmigrantes al salir de las CIEs: indocumentados, sin redes de apoyo y medios para subsistir, situación que los convierte en presa fácil de las redes de tratas de personas, siendo altamente vulnerables a una vida sin derechos.

Lo cierto es que la migración como fenómeno complejo implica a protagonistas de diferentes procedencias y conlleva repercusiones en múltiples contextos, tanto en países receptores como en los de origen y muy en diversas direcciones (Santibáñez, 2006:p.9). La movilidad humana por lo expresado, termina siendo un fenómeno con muchas facetas; es un fenómeno multicausal derivado de las diferentes matices que impulsan a los individuos en la esfera planetaria -en un mundo que dice estar globalizado-a salir casi de manera obligada de sus países originarios, pues ante un capitalismo que devora, la crisis económica europea, y contextos bélicos, por mencionar algunos ejemplos, la subsistencia a toda costa es la constante. Ciertamente, estamos inmersos en una especie de marginalidad avanzada como sugeriría el sociólogo Wacquant (2005, p. 1112), pero no por falta de crecimiento económico sino por todo lo contrario.

2 "Los datos ofrecidos por la Secretaria de Estado de seguridad a través de los informes del Centro de Inteligencia contra el Crimen Organizado, se refieren desde el año 2009 a Rumanía como el primer país de origen de las víctimas de trata con fines de explotación sexual. En 2010 fueron identificadas 366 personas". (Víd. Defensor del Pueblo, 2012, p. 105)
Contexto en el cual aunque los y las inmigrantes terminan siendo los "extraños" objeto de sospecha, lo más paradójico es que aún el grado de vulnerabilidad en el que puedan estar expuestos, por el rechazo en mayor o menor medida, se desconoce que es la inmigración, uno de los aspectos que mueve la economía (Puyol, 2002, p.56), al ser la "comunidad de inmigrantes quienes más aportan al tesoro público" (Aparicio, 2002, p. 145-175), lo cual advierte el rol sustancial que juegan en las sociedades de acogida.

\section{Dos casos concretos: Europa y América Latina}

Hablar de dos contextos relacionados, en este caso, América Latina y Europa, no deja de ser una tarea compleja en temas de inmigración. Dicha cuestión aunque goza de seguimiento, estudio y análisis, es una temática que sigue suscitando interés y muchos interrogantes, entre otras cosas, por el lastre peyorativo con el que suele ser identificada la comunidad de inmigrantes latinoamericana, sin tener en cuenta la ventaja significativa para el país receptor.

La verdad es que en América Latina y el Caribe "cada vez más mujeres migran y se convierten en proveedoras económicas de sus hogares. Este fenómeno ha llevado a que la migración laboral de mujeres para asumir tareas de reproducción en los países de destino, dé lugar a las cadenas globales que se forman a través de la importancia de cuidado y amor de los países pobres a los países ricos" (CEPAL, 2007, p.2). Al respecto, la bibliografía especializada pone en evidencia, en primer lugar, en el caso de Europa (Cfr. el cuadro ilustrado), $y$, específicamente en España, "la elevada concentración sectorial y ocupacional de las trabajadoras extranjeras en nichos de empleo de menor cualificación, más intensa que la que se da entre los hombres extranjeros y entre las mujeres españolas" (Molpeceres, 2012, p. 94). Aunque esta tendencia se observa también en otros países, para el caso de España reviste particularidades propias, asociadas todas ellas con la tercerización, precarización y segmentación del empleo (2012, p. 94). 


\section{Personas que llegaron a la Unión Europea (UE) por subregión de origen de América Latina y el Caribe (ALC) según el género, 1998-2012}

\begin{tabular}{cccc}
\hline Subregión de Origen & Mujeres & Flujo total & $\begin{array}{c}\text { Porcentaje de mujeres con } \\
\text { respecto al flujo total }\end{array}$ \\
\hline América Central & 134.581 & 219.773 & 61 \\
\hline Caribe & 194.367 & 330.510 & 59 \\
\hline América del Sur & 2.801 .635 & 1.539 .241 & 55 \\
\hline Total ALC & 308.145 & 555.023 & 56 \\
\hline Flujo total de entrada a la UE & 45.693 .802 & 20.792 .371 & 46 \\
\hline
\end{tabular}

Tomado de: (OIM, 2015, p. 22)

Estudios más bien recientes (OIM, 2015; Canales, 2013; Martínez, 2011) destacan que la población de origen latinoamericano ha desarrollado un papel muy importante en el intenso crecimiento de los flujos migratorios internacionales en España, al resaltar como innegable el aporte en lo atinente a la dinámica económica y social del país. De hecho, dichos estudios confirman, en relación a la situación demográfica que:

(...) en España la proporción de la población de 65 años y más creció de $10 \%$ en 1970 a $17 \%$ en 2010 . En sentido inverso se observa el comportamiento de la población infantil que pasó de $28 \%$ a $15 \%$ en el mismo periodo. Bajo el contexto de envejecimiento poblacional y bajas tasas de fertilidad se pueden identificar algunos efectos de la crisis demográfica en el mercado laboral: de 1999 a 2012 se generaron en España 3,8 millones de empleos, de los cuales la población nativa económicamente activa ocupó sólo 1,7 millones, creándose un "déficit" de 2,1 millones de personas. El aporte de los migrantes de ALC en este vacío es fundamental, si se considera que en el periodo 2000-2012 poco más de 1,5 millones de trabajadores latinoamericanos y caribeños, de entre 15 y 49 años, se incorporaron a la fuerza laboral española (OIM, 2015).
Finalmente, a la fecha, según el estudio de la OIM (2015), titulado, Dinámicas migratorias en América Latina y el Caribe (ALC) y entre ALC y la Unión Europea, revela que desde 2010 -y por primera vez en 14 años- el flujo de salida desde la UE con destino a ALC fue superior que el flujo de entrada en sentido inverso. En 2012, hubo 181.166 ciudadanos europeos que emigraron a los países de ALC, en comparación con los 119.000 latinoamericanos y caribeños que emigraron a la UE. Esto representa una disminución del $68 \%$ con respecto al año 2007, cuando el número de personas que emigraron de ALC a la UE alcanzó máximos históricos. Todo lo cual es resultado de la actual crisis económica que enfrenta Europa, y muy especialmente España, situación que demuestra que la migración debe ser entendida como un proceso natural, que trae ventajas, contrario a la percepción negativa con la que ha sido entendida en los últimos tiempos, bajo la concepción errónea que los "recién llegados" amenazan la seguridad nacional.

El estudio a su vez, demuestra lo argumentado en este escrito, al considerar que la migración desde ALC hacia la UE está altamente feminizada. En el periodo 1998-2012 el 56\% de las personas que entraron a la UE procedentes de ALC fueron mujeres, siendo la región de América Central la que tiene la proporción más alta $(61 \%)$, (OIM, 2015, p. 6). Nótese que en España "hacia 1999 la inmigración latinoamericana era prácticamente marginal. 
Se registraban tan solo 158 mil trabajadores latinoamericanos trabajando en ese país, los que representaban menos del $1 \%$ de la fuerza de trabajo. Para el 2012, en cambio, la inmigración latinoamericana ya aportaba el $8.6 \%$ de la fuerza de trabajo, e involucraba a un volumen de casi 2 millones de trabajadores" (Canales, 2013, p. 35).

Como se puede observar la presencia femenina en este evento no es la excepción, pero para hacer un análisis menos extensivo, van a tomarse a posteriori dos referentes que servirán como ejemplo para explicar cómo están relacionados los países receptores y emisores de inmigrantes. En este caso, los referentes traídos a contexto en el marco del continente americano y europeo serán dos: por un lado, Colombia y, por el otro, España. La razón de tal cosa es que Colombia (Garay, 2005, p. 37) es uno de los países latinoamericanos con mayor flujo de emigrantes, en cuyo caso, se encuentra España como uno de los principales países Receptores. Además, a diferencia de otros colectivos de inmigrantes que se encuentran muy concentrados en una o dos áreas geográficas, la migración colombiana está más dispersa por toda la geografía española (OIM, 2007, p. 13).

\section{Algunos datos estadísticos en relación a Colombia y España}

Puntualmente, según la OIM en Colombia, el primer periodo de migración masiva hacia España comienza "en la década de los sesenta, coincidiendo con el surgimiento de las guerrillas campesinas, y tuvo a Estados Unidos y Venezuela como destinos principales (...). La oleada migratoria más reciente comienza a gestarse en los inicios de los años noventa, alcanzando su punto más alto en el año 2000" (INSTRAW- OIM, 2007, p. 9). Las principales razones que ocasionan la salida del país, en el caso de Colombia, son de corte económico, seguidas por motivos referidos a la inseguridad, aspectos que no pueden desconocer el legado del conflicto armado.

El carácter novedoso de la emigración colombiana al exterior está también conectado con la feminización de los flujos migratorios por el impacto del envío de remesas tanto a nivel macro como microeconómico. Impacto que es relevante si se toma en cuenta que para el año 2005 el 9\% de la población colombiana se encontraba residiendo en el exterior (Martínez, 2003, p.81). Sin embargo, el rápido incremento de la migración colombiana a España se produce en un contexto de crecimiento general de la migración latinoamericana en ese mismo periodo, lo que evidencia la importancia de los vínculos coloniales e históricos entre España y América Latina y la configuración de España como destino alternativo a Estados Unidos (INSTRAW- OIM, 2007).

En lo que respecta a Colombia, las estimaciones realizadas por el Departamento Administrativo Nacional de Estadística (DANE, Censo 2005), arrojan que es a partir de 1995 cuando se produce un fuerte aceleramiento que hace que en el breve lapso comprendido entre 2000 y 2005, la emigración colombiana crezca casi en un millón de personas (INSTRAW- OIM, 2007: 9). Los datos arrojados confirman que las estimaciones más temerarias respecto al volumen de la migración colombiana a España se quedaron cortas, pues se estima que a noviembre 30 del 2005, se encontraban 779.479 colombianos en España. Según esto, España se convirtió en el segundo en importancia (23.1\%), después de los Estados Unidos (con el 34.6\%), en recepción de emigrantes colombianos, desplazando a Venezuela al tercer lugar (con el $20.0 \%$ ) (Cfr. Posso, 2008).

En todo caso, entre España y Colombia se mantiene una correlación fuerte en términos de atracción en estos fenómenos migratorios, situación relacionada como se observó, con la cercanía persistente por nexos históricos, lo cual también está asociado a la emigración de personas españolas a ALC, por la crisis económica europea que persiste desde el año 2008.

\section{Reflexiones finales}

Con este estudio se plantearon algunas cuestiones de obligada reflexión en torno a la 
migración, que no pueden desconocer que la condición de migrante es, por antonomasia, una situación que deriva en amplio margen de vulnerabilidades. Aspectos que de una u otra manera visibilizan las medidas que se vienen adoptando de manera progresiva no sólo en el contexto europeo sino a nivel global. Estas cuestiones permiten concluir lo estudiado básicamente a partir de aspectos sustanciales que se señalan a continuación:

La migración como fenómeno que muta con el paso tiempo deriva de diferentes factores precipitantes y predisponentes; entendiendo por los primeros aquellos factores que empujan a emigrar, son pues las causas detonantes que incitan la movilidad de un país a otro; y los segundos, pueden ser los factores del entorno que influyen en la concreción del primero.

El discurso del "extraño/enemigo" visto en la persona del inmigrante es una constante en la época actual, aún el consenso generalizado de entender los fenómenos migratorios como parte de la propia naturaleza humana; esto ligado también al auge de la globalización. Lo cual reniega de las ventajas existentes no sólo económicas, sino a nivel demográfico, precisamente por el flujo de entrada y salida de personas que coadyuvan en diversas dinámicas del desarrollo humano.

Entender la inmigración como un problema de avalancha masiva que amenaza las sociedades de acogida es un cliché que desborda serias consecuencias, máxime en el seno de sociedades que manejan discursos altamente estereotipados.

El discurso que con frecuencia vincula a los y las inmigrantes con la delincuencia común, es derivado de políticas gubernamentales que pretenden endurecer las leyes en materia migratoria bajo la excusa de la seguridad nacional, y tal cosa va en detrimento de la opción misma de emigrar.

Las políticas diseñadas también buscan desvirtuar el sentido mismo de emigrar, pues se apuesta por el cierre fronterizo antes que permitir extraños que amenacen a la sociedad.
La comunidad de inmigrantes suele estar asociada con la pobreza, lo cual es un elemento que lleva implícito un cierto clasismo para excluir y estigmatizar a los "Otros" que deben ser expulsados.

No queda duda que las mujeres juegan un rol preponderante tanto en los países de salida como en los de destino. Los indicadores macroeconómicos demuestran una clara relevancia del papel que juegan aquellas en los fenómenos migratorios, tal cosa se explica desde el análisis de las remesas donde las mujeres han tomado amplio protagonismo, con un nivel alto de empoderamiento e independencia.

Se suele desconocer el rol importante que desempeñan los/as inmigrantes, pues sin estos algunas dinámicas propias del desarrollo y la subsistencia humana, en algunas zonas, serían impensables.

Por otra parte, las premisas anteriores, todas en su conjunto, influyen en aspectos tales como:

- El abaratamiento de la mano de obra

- Devaluación del capital humano con trabajos mal remunerados, lo cual no indica necesariamente falta de cualificación de las personas inmigrantes.

- Ante la necesidad de sobrevivir los y las inmigrantes aceptan trabajos que nadie desea y sus cualificaciones pasan a un segundo plano, pues no pueden competir en igualdad de condiciones con los nacionales en los países de acogida.

Estos aspectos visibilizan la necesidad de construir sólidas políticas migratorias que sean más humanas y menos deshumanizante, contemplando la importancia que juegan en estos contextos globalizadores los fenómenos migratorios, que más que un problema, son una ventaja vistos en términos recíprocos, que influyen en el lugar de salida y en el de entrada.

Así pues, es una tarea pendiente la construcción de medidas más garantistas y menos 
excluyentes, esencialmente para intentar contrarrestar las desigualdades existentes a nivel planetario, visibilizadas en las condiciones de pobreza y miseria en la que viven gran parte de la población mundial, siendo una de las causas precipitantes para emigrar bajo la legalidad o indocumentados, en busca de un mejor vivir con dignidad.

\section{Referencias}

Aguilera, R. (2012). "Políticas del multiculturalismo, inmigración y derechos diferenciales en el nuevo contexto global", Revista de Derecho, (11), UNED, pp. 919-949. Recuperado de: <http://e-spacio.uned.es/ fez/eserv.php?pid=bibliuned:RDUNED2012-11-4030\&dsID=Documento.pdf $>$.

Aparicio, R. (2002). "El impacto económico de la inmigración: costos para el Estado y movimiento de consumo y salarios", Cuadernos de trabajo social, (15), pp. 145175.

Blanco, Cristina, (2006). Migraciones. Nuevas movilidades en un mundo en movimiento, Bilbao: Anthropos.

Bonelli, E; Ulloa, M. (coords.)., (2001). Tráfico e inmigración de mujeres en España colombianas y ecuatorianas en los servicios domésticos y sexuales, Madrid: ACSUR,

Campillo, A. (2005). "Ciudadanía y extranjería en la sociedad global", Andrés Pedreño Cánovas y Manuel Hernández Pedreño (Coords.). En La condición inmigrante. Exploraciones e investigaciones desde la Región de Murcia, Universidad de Murcia, pp. $107-124$.

Canales, A. (2013). "Migración y desarrollo en las sociedades avanzadas. Una mirada desde América Latina", Polis. Revista Latinoamericana, vol. 12, no. 35, pp. 85-117.
Castillo, F. (2013). "La migración de cuerpo y mano de obra femenina en Andalucía. El trabajo doméstico: una visión antropológica". F. Javier García Castaño, Nina Kressova (eds.)., en Diversidad cultural y migraciones, Granada: Comares, pp. 6582.

Castles, S; Miller, M. (2004). La era de la migración: movimientos internacionales de población en el mundo moderno. Luís Rodolfo Morán (trad.), México: Universidad Autónoma de Zacatecas.

CEAR (2009). Situación de los centros de internamiento para extranjeros en España. Conversaciones junto al muro. Pau Pérez-Sales (dirc.). Informe Técnico, Comisión Española de Ayuda al Refugiado.

CEPAL (2007). Informe a la X Conferencia Regional de la Mujer de América Latina y el Caribe, LC/G.2361 (CRM.10/8), Quito. Recuperado de <http://www.cepal.org/ mujer/noticias/paginas/4/29404/InformeINSTRAW.pdf>.

Defensor del Pueblo (2012). La trata de seres humanos en España. Víctimas invisibles, Madrid. Recuperado de: <http:// ec.europa.eu/anti-trafficking/sites/antitrafficking/files/human trafficking in spain invisible victims es 2.pdf>. [Consultado septiembre de 2015].

Dijk, T. (2005). "Nuevo racismo y noticias. Un enfoque discursivo". 33- 56. Inmigración, género y espacios urbanos. Los tres retos de la diversidad, Mary Nash, Nuria Benach Rovira y Rosa Tello i Robira (eds.)., Barcelona: Bellaterra.

Fernández, S. (2011). Esclavas sexuales en España. Trata con fines de Explotación sexual de mujeres y niñas Paraguayas 
en España, Propuestas para una Cooperación Internacional Eficaz, Madrid: APRAMP.

Fernández, E. (1998), "Extranjeros en medio de nosotros: lógica de la exclusión y del reconocimiento", en Logos: Anales del seminario de metafísica, (32), pp. 99-120.

Franck, A; y Spehar, A. (2010). La migración laboral de las mujeres en el contexto de la globalización, Bruselas: WIDE.

García Borrego, I. (2003), "Los hijos de inmigrantes como tema sociológico: la cuestión de "la segunda generación", en Anduli: Revista andaluza de ciencias sociales, (3), pp. 27-46.

Gil, S; Agrela Romero, B. (2008). “Un mundo en movimiento contextualización de las migraciones internacionales en Europa y América Latina". En Revista de derecho migratorio y extranjería, pp. 1-28. Visto en: <http://www.flacsoandes.edu.ec/web/ imagesFTP/1264008932.migracion.pdf>

Horvitz, L. M.I. (2012). Seguridad y garantías: derecho penal y procesal penal de prevención de peligros, Revista de Estudios de la Justicia, (16), pp. 99-118.

Instituto de la Mujer (2009). Análisis de la situación laboral de las mujeres inmigrantes. Modalidades de inserción, sectores de ocupación e iniciativas empresariales, Madrid, Ministerio de Igualdad. Visto en: $<$ http://www.inmujer.gob.es/areasTematicas/estudios/serieEstudios/docs/analisisLaborallinmigrantes.pdf>.

INSTRAW- OIM (2007), Género y remesas, Migración colombiana del AMCO hacia España, OIM. Disponible en: <http:// www.humanas.org.co/archivos/InformeColombia-Final-Web.pdf $>$.
Kymlicka, W. (2001). Politics in the Vernacular: Nationalism, culticulturalism and citizenship, Oxford University.

Lucas Martín de, F. (2006). "La ciudadanía para los inmigrantes: una condición de la Europa democrática y multicultural", $R e$ vista de Filosofía, (4), pp. 1-19.

Lucas Martín de, F. (2005). "Nuevas estrategias de estigmatización: el derecho, frente a los inmigrantes", en Mutaciones de Leviatán: legitimación de los nuevos modelos penales, Madrid: Akal, pp. 205-218.

Martínez Pizarro, J. (2003). El mapa migratorio de América Latina y el Caribe, las mujeres y el género, Santiago de Chile, UNFPA.

Martínez Pizarro, J. (Ed) (2011). Migración internacional en América Latina y el Caribe. Nuevas tendencias, nuevos enfoques, Santiago de Chile: CEPAL.

Martínez Escamilla, M. (2013). “Mujeres en el CIE. Género, inmigración e internamiento", Madrid: Tercera Prensa.

Molpeceres Álvarez, L. (2012). "Situación laboral de las mujeres inmigrantes en España", en Cuadernos de Relaciones Laborales, vol. 30, (1), pp. 91-113.

Morcillo Martínez, J. (2012). "Una breve revisión de las teorías de las migraciones desde una perspectiva de género. Mujeres rompiendo estereotipos". pp. 1-25., Manuel Cabrera Espinosa (Coord.), IV Congreso sobre historia de las mujeres, Recuperado de: <http://www.revistacodice.es/publi virtuales/iv congreso mujeres/comunicaciones/MORCILLO.pdf>. [Fecha de consulta, 29 de octubre de 2015]. 
Naïr, S. (2010). La Europa mestiza. Inmigración, ciudadanía y codesarrollo, Barcelona: Círculo de lectores.

Naïr, S. (4 de octubre de 2013). "Morir en Lampedusa", en El País. Visto en: $\quad<$ http://internacional.elpais. com/internacional/2013/10/04/actualidad/1380888322 741511.html >.

Organización Internacional para las Migraciones (OIM), Migración y Género, Fundamentos de Gestión de la Migración, desarrollo de políticas sobre migración, (II). Visto en: http://crmsv.org/documentos/ IOM_EMM_Es/v2/V2S10_CM.pdf. [Fecha de consulta, noviembre 23 de 2015].

Orozco, M. La migración y las remesas hacia América Latina: tendencias, mejores prácticas y experiencias de desarrollo, Caracas: SELA, 2014.

Pérez Orozco, A; Paiewonsky, D; García Domínguez, M. (2008). Cruzando fronteras II: Migración y desarrollo desde una perspectiva de género, Instituto Internacional de Investigaciones y Capacitación de las Naciones Unidas para la Promoción de la Mujer Santo Domingo, UN-INSTRAW.

Petrozziello, Allison J (2003). Género en marcha. Trabajando el nexo Migración-desarrollo desde una perspectiva de género, Guías de aprendizaje, Santo Domingo: ONU Mujeres.

Posso Quiceno, J. (2008). “La migración colombiana hacia España, las redes de hogares trasnacionales y los cambios en las relaciones de género", $A L H I M,(14)$, visto en: <http://alhim.revues.org/2162>. [Consultado el 10 agosto 2015].

Puyol Antolín, R. (2006). "Aspectos demográficos de la inmigración”, Revista cua- trimestral de las Facultades de Derecho y Ciencias Económicas y Empresariales, (69), pp. 15-42.

Puyol Antolín, R. (2002). "La inmigración española: ¿Solución demográfica o económica? Federación de Cajas de Ahorros Vasco-Navarras (eds.)., La evolución demográfica: impacto en el sistema económico y social. (X), pp. 56-66. Recuperado de: <http://www.familiasdealbacete.org/ sites/default/files/userfiles/file/inmigracionsp.pdf>.

Sánchez-Rodas Navarro, C. (Dir.) (2010). Inmigración, mujeres y menores, Laborum ed.

Santibáñez Gruber, R., Oñate, M. (2006). Inmigración: Miradas y reflejos. Historias, identidades y claves de intervención social, Bilbao: Universidad de Deusto.

Sassen, S. (2003a). El espectro de la globalización, Buenos Aires: Fondo de Cultura Económica.

Sassen, S. (2003b). Contrageografías de la globalización. Género y ciudadanía en los circuitos transfronterizos, Cristina Vega Solis y Sandra Gil Araujo (Introd)., Madrid: Traficantes de sueños.

Sassen, S. (2007a). Una sociología de la globalización, María Victoria Rodil, (trad.)., Buenos Aires: Katz.

Sassen, S. (2007b). "La ciudad global: emplazamiento estratégico, nueva frontera". Barcelona 1978-1997. Disponible en: <http://www.macba.cat/PDFs/saskia sassen manolo laguillo cas.pdf>. [Consultado, noviembre de 2015].

Sassen, S. (2006). "La formación de las migraciones internacionales: implicaciones po- 
líticas", RIFP (27), pp. 19-40. Disponible en: <http://e-spacio.uned.es:8080/fedora/ get/bibliuned:filopoli-2006-27-37938CE5086B-6191-7366-5BDBADF72E0E/formacion de migraciones.pdf>.

Serrano-Piedecasas, J.R. (2002). "Los delitos contra los derechos de los ciudadano extranjeros". Patricia Laurenzo Copello (Coord.)., Inmigración y derecho penal. Bases para un debate, Valencia: Tirant Monografías, pp. 309-344.

Silveira Gorski, H. (2002). "Los centros de internamiento de extranjeros y el futuro del Estado de derecho", Mientras Tanto, (83), pp. 93-102.

Terradillos Basoco, J. (2006). "Extranjería, inmigración y sistema penal”. L. Ruíz Rodríguez y M. Rodríguez Mesa, (coord.), en Inmigración y sistema penal: retos y desafíos para el siglo XXI, Madrid: Tirant lo Blanch, pp. 39-68.

Torrado Martín-Palomino, E. (2012). "Las migraciones de menores no acompañados desde una perspectiva de género", en $D_{l}$ LEMATA, (10), pp. 65-84.

Valero Escandell, J. (2005). "Miedo a los inmigrantes", En La ciudad y el miedo. Coloquio de Geografía Urbana., Girona, Universitat de Girona, (ed.), pp. 115-123.

Vargas Llovera, M.D. (1998). "La inmigración africana de venta ambulante: el caso de Alicante". pp. 61-80., Francisco Checa (ed.)., en Africanos en la otra orilla. Trabajo, cultura e integración en la España Mediterránea, Barcelona: Icaria.
Velasco, J.C. (2010). "Dejando atrás las fronteras. Las políticas migratorias ante las exigencias de la justicia global". En Arbor, Ciencia, Pensamiento y Cultura, (744), pp. 585-586.

Vicente Giménez, T. (2005). ¿Qué modelo migratorio propone nuestra normativa legal? Andrés Pedreño Cánovas, Manuel Hernández Pedreño (Coord.), en La condición inmigrante. Exploraciones e investigaciones desde la Región de Murcia, Universidad de Murcia, pp. 200-210.

Vite Pérez, M.Á. (2006). "Estado, globalización y exclusión social”, En política y Cultura, (25), pp. 9-26.

Wacquant, L. (2007). "La estigmatización territorial en la edad de la marginalidad avanzada". Ciências Sociais Unisinos, 43 (3), pp. 193-199.

Wacquant, L. (2005). "Castigar a las parias urbanas". En Oficios terrestres, Universidad Nacional de la Plata, XI, (17), pp. 09-14.

Zaffaroni, E.R. (1994). "Minorías desplazadas, delincuencia y poder punitivo", Cuadernos del Instituto Vasco de Criminología, (7), pp. 83-92.

Zamora, J.A. (2005). "Ciudadanía e Inmigración: Las Fronteras de la democracia", Andrés Pedreño Cánovas y Manuel Hernández Pedreño (Coord.), en La condición inmigrante. Exploraciones e investigaciones desde la Región de Murcia, Universidad de Murcia, pp. 141-158. 